\title{
Invulnerability: A Concept Analysis
}

\author{
Tricia Bernecker \\ DeSales University \\ 2755 Station Avenue, Center Valley, PA, 18034-9568
}

Tel: 610-282-1100Ｅ-mail: tricia.bernecker@desales.edu

\begin{abstract}
The concept of invulnerability is explored from the perspective of nursing, medicine, psychology, sociology, public health, and education. Walker and Avant's framework for concept analysis will be used. Definitions of the concept are presented along with related terms. Antecedents, attributes, and consequences of the concept are discussed in detail. Model, borderline, related, and contrary cases are presented to provide a rich representation of the concept. Empirical referents and implications for nursing are presented.
\end{abstract}

Keywords: Invulnerability, Concept analysis, Nursing

\section{Selection of a Concept}

Nurses are exposed to a host of infectious diseases including, hepatitis B and C, human immunodeficiency virus, meningococcal meningitis and tuberculosis to name a few. US Department of Labor's Occupational Safety and Health Administration and the US Center of Disease control recommend universal precautions for all nurses who work in patient care settings. These precautions include, but are not limited to the use of gloves, gowns, protective eyewear, and needle protective-devices when there is a potential exposure to blood or body fluids. However, not all nurses practice universal precautions consistently to protect themselves from these infectious diseases.

The US Centers for Disease Control and Prevention estimate that there are 380,000 exposure incidents annually from needlestick injuries (Swallow, 2006). In effort to prevent needlestick injuries, various interventions have been initiated to help make nurses safer. Some of these include universal precautions, education, changes in needle practices (e.g., not recapping needles), hepatitis B vaccination, and the engineering inventions of needle-protective devices (Trim, 2004). There are over 1,000 US patents on devices for needlestick safety (McConnell, 2000). Needlestick injuries remain emotionally and financially very costly. Therefore, in the year 2010, with all the available interventions, it is unacceptable that nurses are still subject to needlestick injuries.

The unexpected variable, invulnerability, emerged as this author reviewed the literature on needlestick injuries. Clarke, Rockett, Sloane, and Aiken (2002) addressed compliance with safety devices. The study included, 2287 medical-surgical nurses from 22 hospitals nationwide. Clarke et al. (2002), found that $57 \%$ of the nurses surveyed reported being "somewhat" but not "very" worried about acquiring bloodborne diseases from a needlestick injury. Sixty- two percent reported that they thought about the risk less than once a month. Invulnerability was a variable this author felt needed exploration.

\section{Aim of Analysis}

Walker and Avant's (2005) framework for concept analysis will be used to examine and ultimately define the concept of invulnerability. This analysis will add to the body of nursing knowledge that currently lacks a clear understanding of this concept. The aims of this analysis include (a) identity all possible uses of the concept, (b) identify all antecedents, (c) identify all defining attributes and synthesize definition, (d) identify consequences, (e) construct model, borderline, related, and contrary cases, and (f) identify empirical referents. Implications for nursing will also be addressed.

\section{Identify Uses of Concept}

Initially, the literature was reviewed using the term invulnerable. This author discovered that many of the results led to literature that addressed the concept of vulnerable or vulnerability. It was quickly discovered that invulnerably was not the opposite of vulnerable or vulnerability. The author could not extrapolate meaning for the concept of invulnerably from literature focusing on vulnerable or vulnerability. Cumulative Index to Nursing and Allied Health Literature (CINAHL), Academic Search Elite, and Education Resources Information Center (ERIC) databases were searched utilizing the key term invulnerability. Literature was found in the fields of 
nursing, medicine, psychology, sociology, public health, and education.

Academic Search Elite, an interdisciplinary database that has information from 1975 to the present, yielded 177 articles with the key term invulnerability and decreased to 20 hits when the term was requested in the title. CINAHL database, dating from 1982 to the present, provided 38 articles with the key term invulnerability, and narrowed to 5 when the key term was requested in the title. Lastly, ERIC has data back to 1966 and focuses in the field of education. The keyword invulnerability in this database offered 37 articles and only 5 with the term in the title. Many of the same articles were present in a few of the databases. All of the abstracts were reviewed to identify relevant literature for this concept analysis; however, as the abstracts were read many of articles focused on vulnerability. All the pertinent articles were gathered, read and reread. The search engine, Google, was also utilized and provided 633,000 hits. Many were online dictionary definitions. The author reviewed as much of the identified hits for relevance until the data appeared not to be appropriate for this concept analysis.

Limited definitions of the term invulnerability were found. Many of the articles that focused on the concept of invulnerability did not offer a sound definition of this concept. Many dictionaries were referenced for the term invulnerability. Most defined the adjective, invulnerable, and listed invulnerability under the main term invulnerable as a noun. WordRefernce.com English Dictionary (2008) defined invulnerability as "the property of being incapable of being hurt (physically or emotionally)" (para. 1).

Aalsma, Lapsley, and Flannery (2006), in their research of adolescents and personal fables defined invulnerability as not capable of being harmed or injured. Snyder (1997) defined unique invulnerability as a psychologically protective process that one distorts information so that negative human outcomes are less likely to happen to them than to other people. "People believe that 'Bad things will not happen to me,' thereby displaying a sense of unique invulnerability" (Snyder, p. 197). Snyder further discusses invulnerability as an illusion. Helweg-Larsen (1999) defined optimistic bias and stated this is also called unique invulnerability. Her definition "refers to people's tendency to think their risk is less than that of their peers" (Helweg-Larsen, p. 215).

The authors of the article titled, "Cigarette Smoking Among Young Adults: Integrating Adolescent Cognitive Egocentrism with the Trans-Theoretical Model," defined invulnerability as "impervious to the harms associated with smoking (invulnerable)" (Bright, Mckillop, \& Ryder, 2008, p. 20). Murray and Adam (2001) discussed popular explanations of HIV transmission in gay men and defined inserter invulnerability as the "perception that the insertive partner in anal intercourse is not at risk" (p. 83). Lastly, Hall (2005) studied the perceptions and experiences related to the $9 / 11 / 2001$ attacks on adults living in Southeastern United States. A definition of invulnerability was not provided; however, the author referred to invulnerability as a myth.

Related concepts or terms that are often synonymous or that emerged during the review of literature included, invincible, immune, impregnable, risk-taking, unique invulnerability, optimistic bias, indifference, and risk judgment. As evidenced by the review of identified uses of this concept, nursing does not have a clear definition and one must be synthesized.

\section{Antecedents of the Concept Invulnerability}

Walker and Avant (2005) stated that identifying antecedents is an important step in the analysis of a concept. They stated that antecedents are events or incidents that must occur before the concept. This author reviewed all of the relevant literature to extrapolate antecedents for the concept invulnerability.

\subsection{Age}

"Previous research has established that young people tend to operate with a sense of invulnerability when it comes to risk-taking behavior" (Denscombe \& Drucquer, 1999, p. 195). Age and risk taking has been studied for many years in the literature. It has been suggested that as age increases risk-taking decreases. However, Denscombe and Drucquer (1999) and Millstein and Halpern-Felsher (2002) were not able to provide empirical evidence that as age increases invulnerability decreases. Age may be an antecedent for the concept of invulnerability, but this relationship is not verified to be an inverse relationship. Age must be considered and not excluded because of lack of empirical evidence.

\subsection{Prior Experience/Exposure}

Having a prior experience or exposure to an event may decrease invulnerability. Begley (2006) reported that adults avoid risky behaviors because of previous experiences. Thompson, Kent, Swan, Thomas, and Vrungos (2002) found that gay and heterosexual college students who became infected with a sexually transmitted disease from unprotected sex in the past were less likely to use illusionary control strategies. Millstein and Halpern-Felsher (2002) and Denscombe and Drucquer (1999) discussed having a prior experience or exposure in relation to invulnerability. Based upon the review of literature, this author suggests that having a prior experience 
or exposure has a negative relationship with invulnerability.

\subsection{Habit/Routine}

Individuals may develop a sense of invulnerability when they develop a habit or routine. Sharon Dunn, a healthcare practice leader, reviewed the NASA Space Shuttle Columbia tragedy and related it to hospital staff. There was a sense of invulnerability in NASA relating to all the routine procedures that were in place. This can be related to the nurse who knows where the medication is always located and does not check it, as there is a sense of confidence from this habit or routine (Thrall, 2007). This author believes that having a routine/habit has a positive relationship with invulnerability. However, if a negative consequence results from the routine/habit it becomes a prior experience/exposure and them will have a negative effect on invulnerability.

\subsection{Education}

As knowledge increases invulnerability should decrease. Breheny and Stephens (2004), in their study of why adolescent mothers did not use contraceptives, discussed education as a tool that may be used to decrease perceived invulnerability to pregnancy. However, Begley (2006) reported that the literature demonstrates that educating teens on risky behavior is not effective. The strength of education as an antecedent is not known. However, this author believes it is an antecedent of her synthesized definition of invulnerability. Furthermore, this author proposes that it has a negative relationship; as education increases invulnerability decreases.

\section{Critical Attributes}

Walker and Avant (2005) stated that the critical attributes is the heart of the concept analysis, and these attributes provide the insight to the concept. They further stated that these attributes can emerge over time and as more understanding of the concept evolves so can the attributes. This author, upon synthesis of the definition, felt that two attributes were essential to fully understand the concept of invulnerability.

The first attribute is that it is an illusion to the individual who senses invulnerability. It is an erroneous not true perception of the reality of the situation presented. This illusion of the situation provides the basis for the second attribute, a sense of confidence that no harm will come to self or others. Furthermore, invulnerability is not present at all times, but in given situations. This confidence works in harmony with the illusion that is presented to the individual.

\section{Synthesized Definition of Invulnerability}

The resulting synthesized definition of invulnerability emerged after considering all the antecedent and the critical attributes. Invulnerability is an illusion of confidence that provides the individual with a sense that harm will not come to self or others in a given situation.

\section{Consequences of Invulnerability}

Consequences are the events or outcomes that take place after the concept occurs (Walker \& Avant, 2005). The first consequence is that invulnerability many decrease a person's anxiety. This may be a positive consequence of invulnerability. Thompson et al. (2002) stated “... individuals experience psychological stress or discomfort or discomfort at the prospect that they may be vulnerable to a health risk. Denying or underestimating one's risk can reduce the anxiety created by feeling of personal vulnerability" (p.506). This can be evidenced in theories about victim blame. Blaming the victim is a way to distance oneself from the anxiety of an unpleasant situation. Victim blame is a mechanism to confirm one's own invulnerability (Rape Crisis Information, 2008). Self-harm and harm to others are two negative consequences of invulnerability. If a person perceives invulnerability in a given situation, harm may come to self or others.

\section{Model Case and Additional Cases}

\subsection{Model Case}

A model case is a model exemplar of all antecedents and attributes (Walker \& Avant, 2005). The model case can be factual, from the literature, or constructed by the author. This author constructed all the cases presented.

Diane was a newly registered nurse practicing for only two months in her position as a critical care nurse at a large city hospital. She was feeling comfortable in her position and was getting into a routine in the care of her acutely ill patients. Diane received a physicians order to give $5 \mathrm{mg}$ morphine intravenous to an 18-year-old trauma patient who was having pain in the left scapular area. Diane entered the medication room and signed out a $10 \mathrm{mg}$ vial of morphine. Protocol states that a second registered nurse's signature is needed to dispense narcotics. Diane decided she would just ask for that signature later in the shift when someone was available. She witnessed this practice many times and never questioned it. Diane entered the room and pulled the cap off the 
needle with her teeth. This made her feel like a "real nurse." She administered the 5mg morphine as ordered without checking the patients name band first. Diane was never oriented on the proper use of the needle protective device, so she set the unprotected used needle on the bedside table while she completed the rest of her nursing interventions. During the next shift, a technical partner was stuck by the contaminated needle left on the bedside table.

The above model case includes all the defining attributes critical to the concept of invulnerability. This individual has an illusion of confidence that harm will not come to herself or others. Furthermore, the antecedents of age, habit/routine, and education were present in this case. Prior experience/exposure was not present as this antecedent has a negative relationship with invulnerability. The consequence of harm to others was also present in this exemplar.

\subsection{Borderline Case}

Walker and Avant (2005) discussed how borderline cases help clarify the thinking about the concept. Borderline cases contain most of the critical attributes but not all of them.

Sam has now been practicing as a registered nurse for over one year. He started the day realizing he was assigned to care for the mother of his college roommate, a good friend. She was admitted two days earlier with chest pain, but for unknown reasons has been becoming increasing confused. During report, it was stated that the patient was in restraints to prevent her from pulling out the central venous catheter for the second time. When Sam entered the room, his friend's mother immediately recognized him. She carried on a sound conversation and pleaded with Sam to remove the restraints. She stated, "they make me feel like I am a child in prison." Over the past year, Sam had never had an incident in regards to patient safety, so with slight reluctance he removed the restraints. Two hours later, Sam entered the patient's room and discovered the central venous catheter on the floor and the patient bleeding from the exit site.

The borderline case presented is missing the attribute of confidence. The nurse had slight reluctance to removing the restraints. All the antecedents were present including, age, prior experience/exposure, habit/routine, and education. In this case, the consequence was harm to others.

\subsection{Related Case}

"Related cases are those cases that demonstrate ideas that are very similar to the main concept but differ when examined closely" (Walker \& Avant, 2005, p. 710). These cases are similar but do not contain all of the defining attributes.

Paige was having a horrible day working on the progressive coronary care unit. Two nurses called out sick, so her patient assignment was very challenging. Paige did not even get a chance to assess the patient in room $22 \mathrm{~B}$ when the technical partner interrupted her getting report. The technical partner stated that the patient in 22B was getting a headache and needed Tylenol immediately. Paige stopped the tape recorder that was playing the shift report and went into the medication room. Paige opened the bottom drawer of the medication cart where Tylenol is normally stocked and grabbed a package of medication. Paige went to the bedside of 22B, opened the white package, and handed the patient the two pills without checking the patient's bracelet or the package label. Paige quickly exited the room to get back to report so she would not get too far behind in her care.

This case appears to demonstrate the concept of invulnerability. However, when examined closely this nurse may not have the illusion of confidence that harm will not come to herself or others. This exemplar may not demonstrate the concept of invulnerability. The actions the nurse is taking in this exemplar may demonstrate a nurse who is extremely busy in her patient care.

\subsection{Contrary Case}

The contrary case is a representation of what the concept is not. Again, this can assist with clarifying the concept (Walker \& Avant, 2005).

Tom has been practicing as a registered nurse for over two years in the open-heart unit. He has received over six months of orientation. The open-heart unit had a routine that Tom could not grasp. He was always very nervous in the care he provided to the critically ill patient. He had a constant fear that he was going to make a mistake and unintentionally harm the patient. He had a medication error during his second week on the unit.

The contrary case does not have any of the defining attributes of the concept invulnerability. Furthermore, the antecedents of prior experience/exposure and education are present and both have a negative relationship with invulnerability. 


\section{Empirical Referents}

According to Walker and Avant (2005), empirical referents are classes or categories of the phenomena that demonstrate the occurrence of the concept. "They are also very useful in practice because they provide the clinician with clear, observable phenomena by which to determine the existence of the concept in particular clients" (Walker \& Avant, p. 74). A measurement instrument of invulnerability, from a nursing perspective, has not been developed. Hopefully, the analysis of this concept will assist in the development of an instrument. Morojele, Brook, and Kachieng'A (2006) and Breheny and Stephens (2004) studied risk behaviors and perceived invulnerability qualitatively. Millstein and Halpern-Felsher (2002) quantitatively studied risk judgment. Flin, Yule, McKenzie, Paterson-Brown, and Maran (2006) utilized attitude surveys that indicated a belief in personal invulnerability to stress and fatigue in both nurses and surgeons. Bright et al. (2008) used the New Personal Fable Scale instrument that yields three sub-scale scores for the personal fables of uniqueness, invulnerability, and omnipotence. Hunter (2005) used two Likert-type scales to assess pilots' hazardous attitudes including the factor of invulnerability. All these instruments and study methods discussed attempt to extrapolate a meaning of invulnerability. This author believes it is essential to develop an instrument to measure the concept of invulnerability specifically from a nursing perspective.

\section{Implications for Nursing}

This author believes that the concept of invulnerability is present in nursing and the healthcare setting. The use of universal precautions amongst healthcare workers, patient safety from restraints or falls, medication administration, transcription of doctor orders are a few examples of instances were nurses' perception of invulnerability could have a negative outcome for the nurse or the patient. It is essential that the concept of invulnerability is fully understood, so appropriate interventions can be utilized to protect the healthcare worker and patient.

\section{Conclusion}

Invulnerability was a relatively unknown concept in nursing. The concept analysis utilizing Walker and Avant's framework has provided new insight to this phenomenon. This analysis had added new knowledge to the discipline of nursing. The concept of invulnerability needs to be continued to be explored, developed, and understood.

\section{References}

Aalsma, M., Lapsley, D., \& Flannery, D. (2006). Personal fables, narcissism, and adolescent adjustment. Psychology in the Schools, 43, 481-452.

Begley, S. (2006). Reasoning won't curb teens' risky behavior, scientists say. The Charleston Gazette, 4.

Breheny, M., \& Stephens, C. (2004). Barriers to effective contraception and strategies for overcoming them among adolescent mothers. Public Health Nursing, 21, 220-227.

Bright, S., Mckillop, D., \& Ryder, D. (2008). Cigarette smoking among young adults: Integrating adolescent cognitive egocentrism with the trans-theoretical model. Australian Journal of Psychology, 60, 18-25.

Clarke, S. P., Rockett, J. L., Sloane, D. M., \& Aiken, L. H. (2002). Organizational climate, staffing, and safety equipment as predictors of needlestick injuries and near-misses in hospital nurses. American Journal of Infection Control, 30, 207- 216.

Denscombe, M., \& Drucquer, N. (1999). Critical incidents and invulnerability to risk: Young people's experience of serious health- related incidents and their willingness to take heath risks. Health, Risk \& Society, 1, 195-207.

Flin, R., Yule, S., McKenzie, L., Paterson-Brown, S., \& Maran, N. (2006). Attitudes to teamwork and safety in the operating theatre. Surgeon, 4, 145-151.

Hall, J. (2005). Responses of adults in the southeast to events of September 11: Six months later. Issues in Mental Health Nursing, 26, 415-432.

Helweg-Larsen M. (1999). Students do not overestimate their life expectancy: An alternative demonstration of unique invulnerability. Teaching in Psychology, 26, 215- 217.

Hunter, D. (2005). Measurement of hazardous attitudes among pilots. The International Journal of Aviation Psychology, 15, 23-43.

McConnell, E. A. (2000). Needlestick injuries among health care workers: A literature review - Brief review. [Online] Available: http://articles.findarticles.com/p/articles/mi_m0FSL/is_1_71/ai_59035041 (October 1, 2009). 
Millstein, S., \& Halpern-Felsher, B. (2002). Judgments about risk and perceived invulnerability in adolescents and young adults. Journal of Research on Adolescence, 12, 399-422.

Morojele, N., Brook, J., \& Kachieng'A, M. (2006). Perceptions of sexual risk behaviors and substance abuse among adolescents in South Africa: A qualitative investigation. AIDS Care, 18, 215-219.

Murray, J., \& Adam, B. (2001). Aging, sexuality, and issues among older gay men. The Canadian Journal of Human Sexuality, 10, 75-90.

Rape Crisis Information (2008). Victim blame. [Online] Available: http://www.ibiblio.org/rcip//vb.html\#pagebegin (October 10, 2009).

Snyder, C. (1997). Unique invulnerability: A classroom demonstration in eliminating personal mortality. Teaching in Psychology, 24, 197-199.

Swallow, A. (2006). Cutting down on sharps injuries. OR Insider, November, 6-7.

Thompson, S., Kyle, D., Swan, J., Thomas, C., \& Vrungos, S. (2002). Increasing condom use by undermining perceived invulnerability to HIV. AIDS Education and Prevention, 14, 505-514.

Thrall, T. (2007). Lessons from a NASA tragedy. $H \& H N$ : Hospitals \& Health Networks, 81, LS-5.

Trim, J. C. (2004). A review of needle-protective devices to prevent sharps injuries. British Journal of Nursing, 13, 144-153.

Walker, L. O., \& Avant, K. C. (2005). Strategies for theory construction in nursing ( ${ }^{\text {th }}$ ed.). Norwalk, CT: Appleton \& Lange.

WordReference.com English Dictionary. (2008). Invulnerability. [Online] Available: http://www.wordreference.com/definition/invulnerability (October 10, 2009). 